\title{
OXIDANT/ANTIOXIDANT STATUS ASSESSMENT OF BLOOD IN SELECTED EQUINE DISEASES
}

\author{
WIESŁAW KRUMRYCH, JANUSZ ZBYLUT, MARCIN GOŁYŃSKI ${ }^{1}$, \\ AND HANNA MARKIEWICZ \\ Department of Pathophysiology of Reproduction and Mammary Gland, \\ National Veterinary Research Institute, 85-090 Bydgoszcz, Poland \\ ${ }^{1}$ Department of Internal Diseases of Farm Animals and Horses, \\ Department and Clinic of Animal Internal Diseases, Faculty of Veterinary Medicine, \\ University of Life Sciences, 20-950 Lublin, Poland \\ wieslaw.krumrych@piwet.pulawy.pl
}

Received: November 29, 2012

Accepted: May 2, 2013

\begin{abstract}
The objective of the study was to assess ratio of oxidation to antioxidation in the blood of horses in selected equine diseases. The study was conducted on 47 Polish half-breed horses (36 mares, six stallions, and five geldings) aged 2 to 16 years. Nineteen mares were diagnosed with endometritis, seven horses with symptoms of colic, and six with upper respiratory tract infection. The remaining clinically healthy horses $(n=15)$ served as control. The following parameters were measured: oxygen metabolism in neutrophils using a chemiluminescence (CL) method, total antioxidant status (TAS) of blood plasma, as well as superoxide dismutase (SOD), glutathione peroxidase (GPx), and catalase activity in whole blood. The results demonstrate increased CL of neutrophils in mares with endometritis, as well as in horses with symptoms of colic. This finding was associated with decreased values of SOD, GPx, and TAS (chiefly in mares with endometritis), which suggests oxidative stress. These results underscore the importance of a broader use of oxidant/antioxidant status assessment in studies of the pathogenesis and pathomechanisms of equine diseases.
\end{abstract}

Key words: horses, blood, oxidant status, antioxidant status, diseases.

One of the conditions of homeostasis is to maintain balance between reactive oxygen species (ROS) formation and the antioxidant capacity of the organism. Increased ROS formation and/or attenuation of antioxidant mechanisms lead to so called "oxidative stress" (2). The results of this phenomenon are well documented changes in the conformation of many biological molecules, which lead to abnormalities in their function. The structures most susceptible to ROSmediated damage are the lipids compromising the cell membranes (changes in integrity and fluidity) as well as nucleic acids, proteins, and carbohydrates $(2,7)$. The pathological implications of free radical reactions and oxidative stress are still the core object of much research that suggests an important role of ROS in the pathomechanisms of many diseases with immune dysfunction and chronic inflammation (12). The number of studies concentrating on these problems in horses is undoubtedly lower than those devoted to humans and laboratory animals, however, the observations made so far present increased ROS formation in the course of many diseases, among others: recurrent airway obstruction (4, 11), exercise-induced pulmonary haemorrhage (19), equine motor neuron disease (5), joint diseases (6), laminitis (21), and bacterial endometritis (16). It needs to be emphasised that these studies were limited to the assessment of oxygen metabolism in neutrophils or the antioxidant potential of blood. It seems, however, that a simultaneous assessment of both parameters would provide a better understanding of disturbances in oxidative-antioxidative balance.

The purpose of the study was to assess the oxidative-antioxidative status in the peripheral blood during the course of selected equine diseases.

\section{Material and Methods}

Animals. The study was conducted on 47 Polish half-breed horses (36 mares, six stallions, and five geldings) aged 2 to 16 years; namely, 19 mares with endometritis, which was diagnosed based upon cytologic and bacteriologic assessment of smears taken from the uterus (group I), seven animals (four mares, one stallion, two geldings) with symptoms of mild to moderate colic pain caused by acute gastrointestinal disease (group II), and six horses (three mares, two stallions, one gelding) 
with symptoms of cough and mucopurulent discharge from the nose suggesting inflammation of the upper respiratory tract (group III). The remaining clinically healthy animals (ten mares, three stallions, and two geldings) served as the control group (C).

Sample collection. Blood was taken from the external jugular vein at the moment of diagnosis of the illness (examination A) as well as 2-3 weeks after completion of therapy (examination B). The blood was sampled using a closed negative pressure system Vacuette $^{\circledR}$. Each sample was collected into two plastic tubes: one $9 \mathrm{~mL}$ tube containing lithium heparin and one $4 \mathrm{~mL}$ tube containing potassium versatate $\left(\mathrm{K}_{2}\right.$ EDTA).

Chemiluminescence of peripheral blood neutrophil analysis. The evaluation of oxygen metabolism in neutrophils was done in whole blood (collected into tubes containing heparin) using chemiluminescence (CL) with luminol (5-amino-2,3dihydro-1,4-phthalazinedione) diluted in a $0.4 \%$ solution of $\mathrm{NaOH}$ to a concentration of $28 \mu \mathrm{mol} / \mathrm{mL}$. The assessment was performed using a microplate luminometer (Luminoskan Ascent Type 392, ThermoLabsystems, Finland) by kinetic method for $40 \mathrm{~min}$ at $38.0^{\circ} \mathrm{C} \pm 0.1^{\circ} \mathrm{C}$, measuring $\mathrm{CL}$ at 2 -min intervals. The results were presented as the value of CL integration that is the area under the curve of emission plotted against the function of time (RLU). Both spontaneous luminol dependent (without stimulation WS) and stimulated chemiluminescence was determined. The following CL stimulators were used: opsonised zymosan (OZ), N-formyl-methionyl-leucylphenylalanine (fMLP), and phorbol myristate acetate (PMA). The examined sample contained: $150 \mu \mathrm{L}$ of whole blood, $100 \mu \mathrm{L}$ of $1.41 \mathrm{mmol} / \mathrm{L}$ luminol solution, and $200 \mu \mathrm{L}$ of PBS or $100 \mu \mathrm{L}$ of PBS $+100 \mu \mathrm{L}$ of stimulator. The sample was transferred at $300 \mu \mathrm{L}$ volume to microplate wells using an automatic pipette. The tests started immediately after the addition of blood to the prepared reagents. All measurements were repeated three times during one test and the arithmetic mean value was calculated.

Due to the fact that $\mathrm{CL}$ value is inversely proportional to the concentration of haemoglobin $(\mathrm{Hb})$, whose spectrum of light absorption weakens chemiluminescence, and directly proportional to the number of neutrophils $(\mathrm{N})$ in the sample, the obtained results were corrected relating $\mathrm{CL}$ value to 1,000 cells. In the blood collected into tubes containing $\mathrm{K}_{2}$ EDTA, $\mathrm{Hb}$ concentration was determined, as well as the white blood cell WBC count, and the percentage of neutrophils in WBC picture. Haematological indices (red blood cells, WBC, $\mathrm{Hb}$, haematocrit) were determined using an automatic analyser (ABCVet, Horiba ABX, France) and microscopic analysis of blood smears was performed using May-Grünwald-Giemsa staining. Optimisation of the results was done according to the following formula taking into account the volume of the blood sample (15):

$$
\mathrm{CL} \text { calculated }=\frac{\mathrm{CL} \text { measured } \times \mathrm{Hb}}{\mathrm{SWC} \times \mathrm{N} \times 150-/ 100}
$$

$\mathrm{Hb}$ - percentage of haemoglobin content (in relation to the mean value calculated for the particular group of horses $)$, WBC $\left(\times 10^{3} / \mu \mathrm{L}\right)$ - absolute value of white blood cells count, $\mathrm{N}$ - percentage of neutrophils in WBC picture, 150 - volume of blood in $\mu \mathrm{L}$.

Antioxidative potential of blood analysis. An assay of enzymatic antioxidative potential involved determination of the activity of superoxide dismutase (SOD) in erythrocytes, as well as glutathione peroxidase (GPx) and catalase (CAT) in heparinised whole blood. For this purpose, commercial sets of reagents were used: RANSOD test, RANSEL test (Randox Laboratories Ltd., UK), and BIOXYTECH test Catalase-520 (Oxis Research, USA), respectively. Total antioxidative status (TAS) of blood plasma was determined using Total Antioxidant Status test (Randox Laboratories Ltd., UK). All measurements were performed using spectrophotometer EPOLL-20 (POLL Ltd., Poland) according to the manufacturer's recommendations. Blood samples were analysed twice (during one assay) and an arithmetic mean was calculated. The quality of measurements was verified systematically against controls: RANSOD Control, RANSEL Control, Total Antioxidant Status Control Serum (Randox Laboratories Ltd. UK), and Aspergillus niger catalase (Oxis International Inc., USA). The determined enzymatic activity was converted into $\mathrm{Hb}$ concentration and presented for SOD and GPx as U/g Hb and for CAT as $\mathrm{U} / \mathrm{mg} \mathrm{Hb}$. Total antioxidant status was expressed in $\mathrm{mmol} / \mathrm{L}$.

Statistical analysis. The obtained results are presented as arithmetic mean $(\bar{x})$ and standard deviation $( \pm \mathrm{SD})$. The significance of the differences between mean values was verified using Tukey test assuming the differences to be significant if their probability was below 5\%. All statistical analyses were performed using Statistica v. 6.0 StatSoft software.

\section{Results}

The haematological examinations revealed minor variation of the assessed mean values between the different groups of animals, both at the moment of diagnosis of the disease and after therapy. Only one statistically significant difference was noted in the number of leukocytes in the group of horses with symptoms of colic (II). The mean value of WBC - at the moment of diagnosis was $11.44 \pm 2.93 \mathrm{G} / \mathrm{L}$ compared to $8.50 \pm 1.81 \mathrm{G} / \mathrm{L}$ in the same horses after therapy. These changes were accompanied by a small (statistically insignificant) increase in the percentage of neutrophils (approximately 7\%) in sick animals (at the expense of lymphocyte involvement). In the remaining two experimental groups (I and III) no significant variation in haematological parameters was observed; however, a small variation in WBC indices in mares with endometritis (group I) showed a similar tendency to the horses in group II. It needs to be emphasised that the mean values of the measured haematological indices, in both: the experimental animals (assessments A and B), 
as well as in controls were within the physiological norms of the species (14).

The results of oxidant activity in the blood, assessed by chemiluminescence tests, are shown in Table 1. These indicate a statistically significant increase in the spontaneous oxygen metabolic activity of neutrophils (CL-WS) in horses with endometritis (IA), and symptoms of colic (IIA) compared to the control animals (C). Similar results were obtained following the use of various stimulators (OZ, fMLP, PMA). After the treatment of horses in groups I and II, a significant decrease in mean CL-WS and CL-PMA values was stated. A similar trend was observed in the results assessed by CL-OZ and CL-fMLP in comparison to the results obtained at the time of the diagnosis. On the other hand, a statistically significant increase in CL activity in horses with diseases of the upper respiratory tract (IIIA) was not confirmed in relation to the results obtained after therapy (IIIB), as well as to the values seen in healthy animals (C).

The mean value of antioxidant enzymes (SOD, GPx, CAT) and TAS of blood plasma are shown in Table 2. The statistical analysis revealed significant variation of these indices (with the exception of CAT) only in animals of group I. In mares with endometritis (IA), a noticeable decrease in SOD and TAS was seen in relation to the results after therapy (IB). There was also a significant decrease in GPx activity in comparison to control animals (C). It is worth noting that a similar tendency was seen in horses of group II; this, however, was not confirmed statistically. No significant influence of upper respiratory tract infection (IIIA) on the mean values of the measured antioxidant indices was found.

Table 1

Mean values $(\bar{x} \pm \mathrm{SD})$ of spontaneous and stimulated chemiluminescence (in RLU) in the peripheral blood of horses

\begin{tabular}{crrrrc}
\hline $\begin{array}{c}\text { Group of } \\
\text { horses (n) }\end{array}$ & Sampling & CL-WS & CL-OZ & CL-fMLP & CL-PMA \\
\hline \multirow{2}{*}{ I (19) } & A & ${ }^{\mathrm{ab}} 1,838 \pm 580$ & ${ }^{\mathrm{b}} 4,176 \pm 1,955$ & ${ }^{\mathrm{b}} 2,383 \pm 972$ & ${ }^{\mathrm{ab}} 2,125 \pm 845$ \\
\cline { 2 - 6 } & $\mathrm{B}$ & $842 \pm 467$ & $3,099 \pm 1,308$ & $1,692 \pm 1,265$ & $1,185 \pm 452$ \\
\hline \multirow{2}{*}{ II (7) } & $\mathrm{A}$ & $\mathrm{a} 1,872 \pm 572$ & $3,749 \pm 1,922$ & ${ }^{\mathrm{b}} 2,216 \pm 718$ & ${ }^{\mathrm{ab}} 2,520 \pm 1,024$ \\
\hline \multirow{2}{*}{ III (6) } & $\mathrm{B}$ & $709 \pm 250$ & $2,613 \pm 1,081$ & $1,195 \pm 511$ & $1,109 \pm 437$ \\
\cline { 2 - 6 } & $\mathrm{A}$ & $787 \pm 233$ & $3,096 \pm 1,529$ & $977 \pm 182$ & $1,275 \pm 218$ \\
\hline C $(15)$ & $\mathrm{B}$ & $626 \pm 193$ & $2,215 \pm 813$ & $914 \pm 201$ & $1,119 \pm 280$ \\
\hline
\end{tabular}

$\mathrm{n}$ - number of horses; I - endometritis; II - symptoms of colic; III - diseases of the upper respiratory tract; C - clinically healthy control horses.; A - assessment at the moment of diagnosis of the disease; B - assessment after therapy; CL-WS - chemiluminescence without stimulation; CL-OZ - stimulated chemiluminescence opsonised zymosan; CL-fMLP stimulated chemiluminescence N-formyl-methionyl-leucyl-phenylalanine; CL-PMA - stimulated chemiluminescence phorbol myristate acetate; ${ }^{a}$ - mean value differs significantly $(\mathrm{P}<0.05)$ compared to value registered in $\mathrm{B}$ assay; ${ }^{\mathrm{b}}$ mean value differs significantly $(\mathrm{P}<0.05)$ compared to value registered in control horses $(\mathrm{C})$

Table 2

Mean values $(\bar{x} \pm \mathrm{SD})$ of antioxidant enzymes activity and total antioxidant status (TAS) in the peripheral blood of horses

\begin{tabular}{cccccc}
\hline $\begin{array}{c}\text { Group of } \\
\text { horses (n) }\end{array}$ & Sampling & $\begin{array}{c}\text { SOD } \\
(\mathrm{U} / \mathrm{g} \mathrm{Hb})\end{array}$ & $\begin{array}{c}\text { GPx } \\
(\mathrm{U} / \mathrm{g} \mathrm{Hb})\end{array}$ & $\begin{array}{c}\text { CAT } \\
(\mathrm{U} / \mathrm{mg} \mathrm{Hb})\end{array}$ & $\begin{array}{c}\text { TAS } \\
(\mathrm{mmol} / \mathrm{L})\end{array}$ \\
\hline \multirow{2}{*}{$\mathrm{I}(19)$} & $\mathrm{A}$ & ${ }^{\mathrm{a}} 2,297 \pm 850$ & ${ }^{\mathrm{b}} 52 \pm 30$ & $445 \pm 107$ & $0.58 \pm 0.14$ \\
\cline { 2 - 6 } & $\mathrm{B}$ & $3,288 \pm 718$ & $77 \pm 24$ & $445 \pm 93$ & $0.8 \pm 0.11$ \\
\hline \multirow{2}{*}{ II (7) } & $\mathrm{A}$ & $2,103 \pm 572$ & $68 \pm 24$ & $522 \pm 111$ & $0.57 \pm 0.19$ \\
\cline { 2 - 6 } & $\mathrm{B}$ & $2,958 \pm 634$ & $79 \pm 22$ & $503 \pm 109$ & $0.75 \pm 0.1$ \\
\hline \multirow{2}{*}{ III (6) } & $\mathrm{A}$ & $2,821 \pm 772$ & $80 \pm 30$ & $413 \pm 104$ & $0.64 \pm 0.17$ \\
\hline & $\mathrm{B}$ & $2,508 \pm 576$ & $73 \pm 25$ & $453 \pm 71$ & $0.73 \pm 0.12$ \\
\hline $\mathrm{C}(15)$ & & $2,872 \pm 550$ & $88 \pm 27$ & $493 \pm 124$ & $0.67 \pm 0.16$ \\
\hline
\end{tabular}

SOD - superoxide dismutase; GPx - glutathione peroxidase; CAT - catalase; n, I, II, III, K, A, B - symbols as in Table 1; ${ }^{a}$ - mean value differs significantly $(\mathrm{P}<0.05)$ compared to value registered in $\mathrm{B}$ assay; ${ }^{\mathrm{b}}$ - mean value differs significantly $(\mathrm{P}<0.05)$ compared to value registered in control horses $(\mathrm{C})$ 


\section{Discussion}

Chemiluminescence in whole blood was analysed on the basis of the assumptions of Magnusson and Holst (18) stating that measurements in whole blood are a better reflection of homeostasis in vivo than examinations performed on isolated leukocytes and are thus more useful in clinical trials. Measurements in whole blood also do not require any laboratory procedures to isolate neutrophils, which could affect their survival, or expression and activity of cell surface receptors (23). It seems, therefore, that methods used in the study (using a set of stimulators) allowed us to obtain objective results, under the assumption that total CL of the blood corresponds to the amount of ROS generated by neutrophils, which are the dominant population of phagocytes in the blood. This is grounded in research presenting that the contribution of monocytes to light generated in test tubes is negligible due to their small numbers and weaker generation of ROS in comparison to neutrophils (17).

Presented results demonstrate intensification of free radical dependent process in the blood of sick horses, especially mares with endometritis and in animals with symptoms of colic. This phenomenon manifested as an increase in both spontaneous and stimulated chemiluminescence activity in blood neutrophils in comparison to the results obtained after therapy and to the results of control animals. The study assessed not only spontaneous CL in the blood but also after its activation with various stimulators. This method is often used in the research because it allows not only for an indirect assessment of the neutrophil ability to generate ROS but also to assess their ability to correct response to stimulation via different signalling mechanisms. Such an experimental setup enables a more complete insight into the functional activity of neutrophils through an evaluation of the expression and distribution of surface receptors and enzymes essential for proper and effective phagocytosis. These results are consistent with the results of other studies. Asbury and Hansen (1) have shown a significant increase in oxygen dependent bactericidal activity of circulating neutrophils in mares with bacterial endometritis compared to healthy animals. Additionally, a similar relationship was documented in the CL of cells of uterine origin. These observations are exceptionally important because they confirm the usefulness of assessing the intensity of ROS generation by primed cells of the peripheral blood in situations involving localised inflammatory states. Similarly, the previous research undertaken in the Department of Pathophysiology of Reproduction and Mammary Gland has shown a positive correlation between CL in the blood and intensity of endometrial inflammation in mares (16).

An intensification of free radical processes in blood was also seen in horses with symptoms of colic. These results overlap with those of previous research. Activation of neutrophils has been observed in strangulating lesions (8) and in inflammatory conditions of the gastrointestinal tract (29). Many gastrointestinal diseases in horses have been found to be characterised by transmural penetration of bacterial endotoxins from the intestinal lumen into the bloodstream, and initiation of a systemic inflammatory response with activation of peripheral blood leukocytes $(28,29)$. Ischaemia and reperfusion processes also occur during colic in horses and an increased release of ROS after resolution of an intestinal torsion for example could be expected. In experimentally induced ischaemia-reperfusion of the jejunum and large colon of horses, significant oxidant/antioxidant disorders were detected in the jejunum (13).

In contrast, there were no significant changes of $\mathrm{CL}$ in horses with inflammation of the upper respiratory tract. These results are difficult to interpret. Although the studies have shown that viruses, among others influenza virus, can activate the respiratory burst, i.e. an increased generation of superoxides by phagocytic cells (24), it needs to be taken into account that infections in the upper respiratory tract could have been caused by non viral factors such as $e . g$. allergens.

The results of many studies have shown that activated phagocytic cells, both migrating cells, as well as those at the focus of inflammation, are the main cellular source of ROS in inflammatory states (2). It is considered, however, that cells of the peripheral blood (mainly neutrophils) are also a good model for research because of their earlier priming e.g. by cytokines released from activated mononuclear cells (3). Recently, there has been an ever increasing body of the research concerning the mutual interdependencies of inflammatory mediators, cytokines, and ROS. These studies have shown, among others, the damaging effects of ROS on the platelet-activating factor (PAF) inactivating hydrolase, which in consequence furthers the inflammatory state (27), and in turn corresponds to other observations indicating a positive correlation between ROS generation and the advancement of the inflammatory state $(10,16)$.

For the purpose of maintaining homeostasis, aerobic organisms have developed, in the course of evolution, intra and extracellular mechanisms to detoxify ROS. This function is carried out by antioxidant compounds, defined as compounds that slow or prevent the oxidation of a given substrate at relatively lower concentrations (2). A critical role in these processes is ascribed to, among others, SOD, GPx, CAT, and nonenzymatic antioxidants such as $\alpha$-tocopherol, ascorbic acid, $\beta$-carotene, reduced glutathione, uric acid, bilirubin, and albumins (2, 26, 31). All substances mentioned above (and many others) mutually interact with each other, creating a complex system of antioxidative protection in the organism. A deficit of any of the elements of this system can cause a decrease in the total antioxidant potential, a consequence of which is oxidative stress. The results of the study suggest that this phenomenon is revealed primarily in mares with endometrial inflammation. This is indicated by an increase in CL activity, which accompanies a clear decrease in SOD and GPx activity, as well as decreased TAS in the blood. These results are in the agreement with prior publication by Yaralioglu-Gurgoze et al. (30), who have shown a significant decrease in erythrocytic 
GPx in mares with endometritis in comparison to healthy animals. This phenomenon was associated with a clear increase in malonyl dialdehyde (MDA) content, which is one of the final products of the peroxidation of lipid cellular membranes, which additionally confirms an inadequate elimination of ROS by antioxidant compounds. Similar results indicating the phenomenon of oxidative stress in association with endometritis have been obtained in women (25), cows (9), and camels (20).

The study suggests that oxidative stress can also appear in horses with symptoms of colic. This is indicated by lower than average values of SOD, GPx, and TAS in comparison to results obtained in these animals after therapy and to healthy control horses, even though this was not confirmed statistically. This observation seems to be valid, especially in the light of recently published results indicating a marked increase in urinary isoprostane content in horses with symptoms of colic (22). These compounds are generated as a result of nonenzymatic free radical peroxidation of polyunsaturated fatty acids (mainly arachidonic acid), and therefore, their increase in biological samples is a sensitive indicator of oxidative stress. It seems that the results of the study could be affected to a large degree not only by a small sample size from the horses with colic $(n=7)$ but also by the variation in the duration and severity of the disease.

The research undertaken indicates that the postulated involvement of ROS in the course of many diseases is justified; however, it is still unclear whether oxidative stress is one of the causes or a consequence of this phenomenon. Moreover, it also suggests a need for a wider use of oxidant-antioxidant status assessment in studies of the pathogenesis and pathomechanisms of many equine diseases, also in the context of using medicines, which modulate the bactericidal activity of neutrophils and exogenous antioxidant compounds.

\section{References}

1. Asbury A.C., Hansen P.J.: Effects of susceptibility of mares to endometritis and stage of cycle on phagocytic activity of uterine-derived neutrophils. J Reprod Fert 1987, 35, 311-316.

2. Bartosz G.: Druga twarz tlenu. Wolne rodniki w przyrodzie. Edited by PWN, Warszawa, 2004, pp. 99239.

3. Benbarek H., Deby-Dupont G., Deby C., Serteyn D.: Direct stimulation of the oxidative activity of isolated equine neutrophils by TNF-alpha and IL-1beta. Vet Immunol Immunopathol 2008, 121, 101-106.

4. Deaton C.M., Marlin D.J., Deaton L., Smith N.C., Harris P.A., Schroter R.C., Kelly F.J.: Comparison of the antioxidant status in tracheal and bronchoalveolar epithelial lining fluids in recurrent airway obstruction. Equine Vet J 2006, 38, 417-422.

5. Delguste C., De Moffarts B., Kirschvink N., Art T., Pincemail J., Defraigne J.O., Amory H., Lekeux P.: Change in blood antioxidant status of horses moved from a stable following diagnosis of equine motor neuron disease. Can Vet J 2007, 48, 1165-1167.
6. Dimock A.N., Siciliano P.D., McIlwraith C.W.: Evidence supporting an increased presence of reactive oxygen species in the diseased equine joint. Equine Vet $\mathrm{J}$ 2000, 32, 439-443.

7. Evans P., Halliwell B.: Micronutrients: oxidant/ antioxidant status. Br J Nutr 2001, 85, 67-74.

8. Grulke S., Benbarek H., Caudron I., Deby-Dupont G., Mathy-Hartert M., Farnir F., Deby C., Lamy M., Serteyn D.: Plasma myeloperoxidase level and polymorphonuclear leukocyte activation in horses suffering from large intestinal obstruction requiring surgery: preliminary results. Can J Vet Res 1999, 63, 142-147.

9. Hanafi E.M., Ahmed W.M., Sherein I., Moez A.E., Khadrawy H.H.E., Hameed A.E.: Effect of clinical endometritis on ovarian activity and oxidative stress status in Egyptian Buffalo-Cows. American-Eurasian J Agric Environ Sci 2008, 4, 530-536.

10. Kimura S., Yonemura T., Kaya H.: Increased oxidative product formation by blood polymorphonuclear leukocytes in human periodontal disease. J Periodont Res 1993, 28, 197-203.

11. Kirschvink N., Smith N., Fiévez L., Bougnet V., Art T., Degand G., Marlin D., Roberts C., Genicot B., Lindsey P., Lekeux P.: Effect of chronic airway inflammation and exercise on pulmonary and systemic antioxidant status of healthy and heaves-affected horses. Equine Vet J 2002, 34, 563-571.

12. Kirschvink N., De Moffarts B., Lekeux P.: The oxidant/antioxidant equilibrium in horses. Vet J 2008, 177, 178-191.

13. Kooreman K., Babbs C., Fessler J.: Effect of ischemia and reperfusion on oxidative processes in the large colon and jejunum of horses. Am J Vet Res 1998, 59, 340-346.

14. Krumrych W.: Wskaźniki laboratoryjne krwi koni wartości referencyjne i interpretacja. National Veterinary Research Institute, Pulawy, Poland 2003, pp. 12-14.

15. Krumrych W., Wiśniewski E.: The influence of selected stimulators on chemiluminescence of peripheral blood neutrophils in horses. Med Weter 2006, 62, 204-206.

16. Krumrych W., Danek J.: Chemiluminescence of peripheral blood neutrophils in mares with endometritis. Bull Vet Inst Pulawy 2012, 56, 51-56.

17. Lojek A., Ciz M., Marnila P., Duskova M., Lilius E.M.: Measurement of whole blood phagocyte chemiluminescence in the Wistar rat. J Biolum Chemilum 1997, 12, 225-231.

18. Magnusson U., Holst H.: Assaying granulocyte phagocytosis by chemiluminescence: Effect of storage time and temperature of blood samples. J Vet Med B 1998, 45, 217-222.

19. Mills P.C., Higgins A.J.: Oxidant injury, nitric oxide and pulmonary vascular function: implications for the exercising horse. Vet J 1997, 153, 125-148.

20. Mohamed H.E.: Antioxidant status and the degree of oxidative stress in dromedary (Camelus dromedaries) with or without endometritis. Vet Res 2008, 2, 1-2.

21. Neville R.F., Hollands T., Collins S.N., Keyte F.V.: Evaluation of urinary TBARS in normal and chronic laminitic ponies. Equine Vet J 2004, 36, 292-294.

22. Noschka E., Werre S.R., Crisman M.V., Thatcher C.D., Milne G.L., Dahlgren L.A.: Implications of urine F2isoprostane metabolite concentration in horses with colic and its potential use as a predictor for surgical intervention. Equine Vet J 2011, 43, 34-41.

23. Papp Z., Smits J.E.G.: Validation and novel applications of the whole-blood chemiluminescence assay of innate immune function in wild vertebrates and domestic chickens. J Wildlife Dis 2007, 43, 623-634. 
24. Peterhans E.: Oxidants and antioxidants in viral diseases - metabolic regulation and autotoxicity. In: Natural Antioxidants in Human Health and Disease, edited by B. Frei, Academic Press, San Diego, 1994, pp. 489-514.

25. Safronova V.G., Matveeva N.K., Maltseva V.N., Kasabulatov N.M., Avkhacheva N.V., Van'ko L.V., Sukhikh G.T.: Regulation of reactive oxygen species production by peripheral blood neutrophils from women with postpartum endometritis. Bull Exp Biol Med 2005, 140, 205-208.

26. Splettstoesser W.D., Schuff-Werner P.: Oxidative stress in phagocytes - "The enemy within". Microsc Res Tech 2002, 57, 441-455.

27. Triggiani M., De Marino V., Sofia M., Faraone S., Ambrosio G., Carratu L., Marone G.: Characterization of platelet-activating factor acetylhydrolase in human bronchoalveolar lavage. Am J Respir Crit Care Med 1997, 156, 94-100.

28. Weiss D.J., Evanson O.A.: Evaluation of lipopolysaccharide-induced activation of equine neutrophils. Am J Vet Res 2002, 63, 811-815.

29. Weiss D.J., Evanson O.A.: Evaluation of activated neutrophils in the blood of horses with colic. Am J Vet Res 2003, 64, 1364-1368.

30. Yaralioglu-Gurgoze S., Cetin H., Cen O., Yilmaz S., Atli M.O.: The changes of malondialdehyde levels and glutathione peroxidase activity in purebred Arabian mares with endometritis. Vet J 2005, 170, 135-137.

31. Yu B.P.: Cellular defenses against damage from reactive oxygen species. Physiol Rev 1994, 74, 139-162. 